\title{
ACCRETION DISK FLARES IN ENERGETIC RADIATION FIELDS
}

\author{
R.F. VAN OSS, G.H.J. VAN DEN OORD and M. KUPERUS \\ Sterrekundig Instituut, P.O.Box 80000, 3508 TA Utrecht, The Netherlands
}

September 17, 1992

\begin{abstract}
A bstract. We consider the physics of magnetic flares in the energetic radiation field of an accretion disk corona (ADC). The X-ray emission from these flares is thought to be responsable for the observed hard powerlaw component in the X-ray spectra of galactic black hole candidates in their 'high' spectral state. During the flare event (inverse Compton) scattering of soft photons from the underlying disk into hard photons occurs on accelerated electrons in current sheets. The electrons are decelerated by the radiation drag force that results from the up-scattering. This friction-like effect of the intense background radiation field on the motion of the electrons in the sheet can be considered as a form of resistivity in the magnetohydrodynamical picture of the current sheet: Compton resistivity. A spectrum is derived for the up-scattered radiation from current sheets in the ADC and it is found that this spectrum mimics a powerlaw above a critical photon energy.
\end{abstract}

Key words: accretion disks - particle acceleration - magnetic fields - inverse Compton scattering

\section{Introduction}

The hard X-ray component in the X-ray spectra of black hole candidates, like Cygnus X-1, is still not satisfactory explained (Ebisawa 1991), but the highly variable nature of this emission however suggests a flare-like mechanism. The presence of magnetic fields in the disk through dynamo-action results, because of the buoyant nature of strong fields in the disk, in a magnetically structured accretion disk corona (Galeev et al 1979). It can be expected that the energy release in the corona is provided by the flaring of (force-free) coronal structures resulting from instabilities triggered by the shearing disk flow. (Aly 1990; Kuijpers 1989). During the flare reconnection of fieldlines relaxes the magnetic topology to a lower energy state while the energy difference is dissipated in current sheets.

\section{Compton Resistivity in Current Sheets}

We have investigated the process of driven magnetic reconnection in neutral sheets (Sweet-Parker type reconnection) in the ADC and considered the resistive processes that operate there. Compared to classical resistivity and anomalous resistive processes, it turns out that the main deceleration of the electrons, that are accelerated by a direct electric field in the sheet, results from the radiation drag force (van Oss et al 1992). This force is a consequence of the directed momentum loss caused by the inverse Compton scattering of soft X-ray photons from the disk into beamed hard $\mathrm{X}$-rays along the electron motion. We have obtained an expression for this radiation force in the realistic case of a half-isotropic radiation field, that is emitted by an infinite plane:

$$
\begin{aligned}
f_{\|} & =-\frac{4}{3} \sigma_{\mathrm{T}} U_{\mathrm{rad}} \gamma^{2}\left[\beta+\frac{3}{8}\left(1+\beta^{2}\right) \sin \alpha\right], \\
f_{\perp} & =\frac{1}{2} \sigma_{\mathrm{T}} U_{\mathrm{rad}} \cos \alpha .
\end{aligned}
$$


Here, $\beta=v / c, \gamma=\left(1-\beta^{2}\right)^{-1 / 2}, U_{\text {rad }}$ is the energy density of the unscattered radiation field and $\alpha$ is the angle between the electron velocity and the emitting plane, positive when the electron moves towards the plane.

Electrons that enter the sheet are accelerated by the electric field until they reach an asymptotic speed, or Lorentz factor $\gamma_{\infty}$, when the radiation force balances the electric force. We assume that in a stationairy situation the bulk of the energetic electrons in the sheet have this constant speed and the resistivity in the sheet is then governed by Compton resistivity. The dissipated magnetic energy then goes directly into up-scattering of photons instead of heating.

\section{Spectrum of Scattered Photons}

We have calculated the spectrum of photons that result from the scattering of a thermal photon distribution emitted by an infinite plane, on an electron moving with an oblique angle $\alpha$ relative to the plane. The energy of the electron is taken to be larger then the typical unscattered photon energy in order to neglect recoil effects. We assumed that the Lorentz factor of the electrons and also the number of scattering electrons in the ADC between $r$ and $r+d r$ are powerlaw functions on radial distance. The spectrum of the radiation $j_{A D C}$ produced by the whole corona can then be obtained by integration resulting in:

$$
j_{\mathrm{ADC}}=x^{-\nu} \gamma(1+\nu, x) \text {. }
$$

Here $x=\epsilon / \epsilon_{1}, \epsilon_{1}=2 k T_{1} \gamma_{1}^{2}(1+\cos \alpha$ ) (subscript ' 1 ' denotes values at the inner edge of the disk corona); $T$ is the temperature of the disk radiation and $\gamma(a, b)$ is the incomplete gamma function; for $x>\nu$ the gamma function approaches a constant value and the $\mathrm{ADC}$ spectrum resembles a powerlaw.

\section{Conclusions}

The theoretically obtained spectrum of the hard X-rays produced during flares in the ADC fits a powerlaw above a certain critical energy. If this value is less or equal then the energy above which the powerlaw can be distinguished in the $\mathrm{X}$ ray spectra of black hole candidates $(\simeq 10 \mathrm{keV})$, this mechanism can explain the spectral component. An analysis of the expected variability from these flares is then needed to compare it with the observed powerspectrum.

\section{References}

Aly, J.J., 1990, Quasi-static evolution of a force-free magnetic field, Comp.Phys.Comm. 59, 13 Ebisawa, K. 1991, Spectral study of black hole candidates observed with Ginga, Ph.D.Thesis, ISAS RN 483, Japan

Galeev, A.A., Rosner, R., Vaiana, G.S., 1979, Structured coronae of accretion disks, ApJ 228,318 Kuijpers, J., 1989, Magnetic flares in close binaries, in Active Close Binaries, ed. Tbanoglu, C., Yavus, I., NATO ASI

van Oss, R.F., van den Oord, G.H.J., Kuperus, M., 1992, Accretion disk flares in energetic radiation fields, submitted to A\&A 